\title{
Study of spontaneous fission lifetimes using nuclear density functional theory
}

\author{
Jhilam Sadhukhan ${ }^{1,2, a, b}$, Katarzyna Mazurek ${ }^{1,2,3}$, and Witold Nazarewicz ${ }^{1,2,4}$ \\ ${ }^{1}$ Department of Physics and Astronomy, University of Tennessee, Knoxville, Tennessee 37996, USA \\ ${ }^{2}$ Physics Division, Oak Ridge National Laboratory, PO Box 2008, Oak Ridge, Tennessee 37831, USA \\ ${ }^{3}$ The Niewodniczanski Institute of Nuclear Physics - PAN, PL-31-342 Krakow, Poland \\ ${ }^{4}$ Institute of Theoretical Physics, Faculty of Physics, University of Warsaw, ul. Hoża 69, \\ 00-681 Warsaw, Poland
}

\begin{abstract}
The spontaneous fission lifetimes have been studied microscopically by minimizing the collective action integral in a two-dimensional collective space of quadrupole moments $\left(\mathrm{Q}_{20}, \mathrm{Q}_{22}\right)$ representing elongation and triaxiality. The microscopic collective potential and inertia tensor are obtained by solving the self-consistent HartreeFock-Bogoliubov (HFB) equations with the Skyrme energy density functional and mixed pairing interaction. The mass tensor is computed within the perturbative Adiabatic TimeDependent HFB (ATDHFB) approach in the cranking approximation. The dynamic fission trajectories have been obtained by minimizing the collective action using two different numerical techniques. The values of spontaneous fission lifetimes obtained in this way are compared with the static results.
\end{abstract}

\section{Introduction}

The spontaneous fission of a nucleus is a many-body quantum tunneling in a multi-dimensional space of nuclear collective coordinates. To explore this large-amplitude collective motion (LACM) microscopically, we employ the ATDHFB theory that provides a consistent theoretical framework to study LACM [1]. In this approach, the collective nuclear dynamics is considered to be much slower than the single particle motion of individual nucleons. This approximation is fulfilled for spontaneous fission where excitation energy of the system is small compared to the fission barrier height $[1,2]$.

The main ingredients for a theoretical calculation of fission lifetime are the collective potential and collective inertia tensor. For heavy nuclei, the microscopic calculation of these two input quantities can be done suitably by using the self-consistent density functional theory (DFT) [3]. Within this approach, based on a suitable energy density functional, constrained HFB equations are solved to obtain the potential energy surface. In the ATDHFB approach, the collective inertia tensor is then obtained

\footnotetext{
ae-mail: jhilam@utk.edu

${ }^{\mathrm{b}}$ On leave of absence from VECC, Kolkata, India.
}

This is an Open Access article distributed under the terms of the Creative Commons Attribution License 2.0, which permits unrestricted use, distribution, and reproduction in any medium, provided the original work is properly cited. 


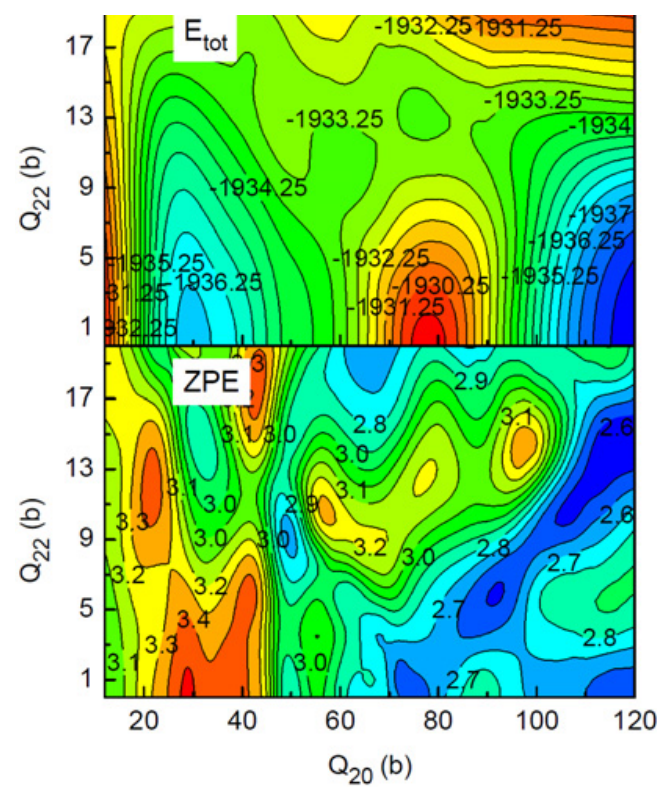

Figure 1. $E_{t o t}$ and $\mathrm{ZPE}$ in $\mathrm{MeV}$ for ${ }^{264} \mathrm{Fm}$ calculated in this work in the collective space of $\left(\mathrm{Q}_{20}, \mathrm{Q}_{22}\right)$.

consistently from the self-consistent densities [3]. These quantities are used in the present paper to study the spontaneous fission half-life by minimizing the collective action integral in a two-dimensional collective space of quadrupole moments $\mathrm{Q}_{20}$ (elongation) and $\mathrm{Q}_{22}$ (triaxiality). In the present study, we have considered ${ }^{264} \mathrm{Fm}$ as the fissioning system and, therefore, we have not considered $\mathrm{Q}_{30}$ (massasymmetry) since symmetric fission is the major fission-decay mode for Fm isotopes.

The theoretical model is outlined in Section 2. Section 3 explains the numerical technique to obtain the minimum action path. The results are presented in Section 4. Finally, the results of this study are summarized in Section 5.

\section{Calculation of energy surface and mass parameters}

The symmetry-unrestricted DFT solver HFODD [4] is used to calculate the collective potential. The Skyrme energy density functional with $\mathrm{SkM}^{*}$ parameterization [5], which is optimized for fission barrier height of ${ }^{240} \mathrm{Pu}$, is employed in the particle-hole channel. In the particle-particle channel, the densitydependent mixed pairing interaction is considered. A detailed description of the input used is given in Ref. [6]. The potential energy surface is obtained in the collective space of $\left(Q_{20}, Q_{22}\right)$ by subtracting the zero-point energy (ZPE) from the total HFB energy $E_{t o t}$. For the present purpose, ZPE is obtained by using the Gaussian overlap approximation [7]. The behavior of $E_{\text {tot }}$ and ZPE for ${ }^{264} \mathrm{Fm}$ is illustrated in Figure 1.

The components of collective inertia tensor in two-dimension are calculated within the perturbative cranking approximation of ATDHFB formula. The expression for the mass tensor reads [3, 7]

$$
M^{p C}=\left[M^{(1)}\right]^{-1} M^{(3)}\left[M^{(1)}\right]^{-1},
$$




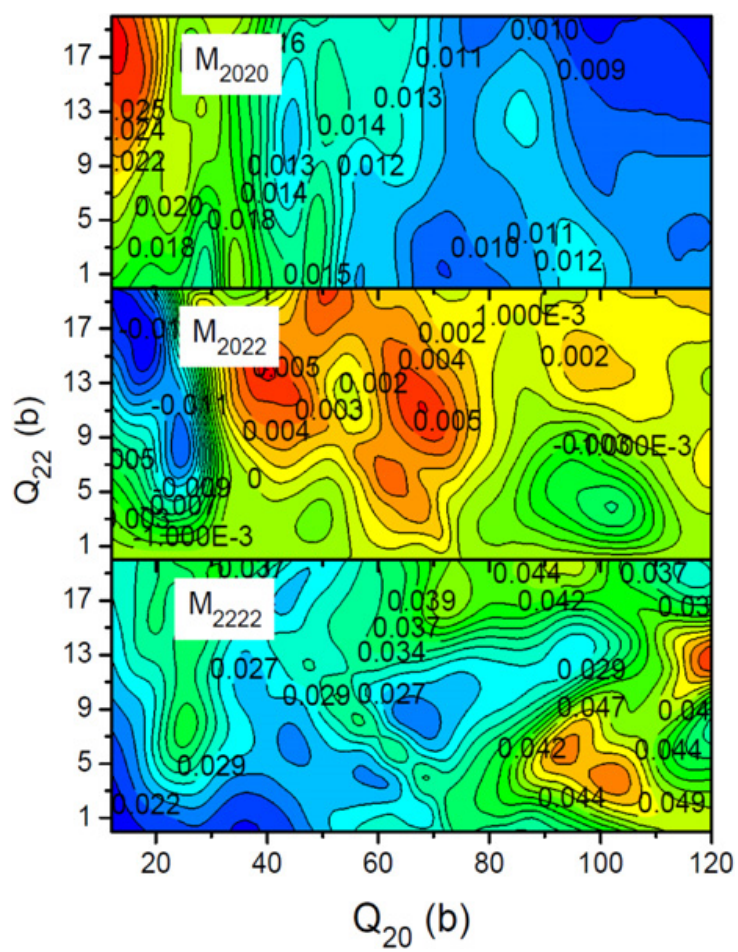

Figure 2. Similar as in Figure 1 but for the perturbative cranking inertia $M^{p C}$.

where the energy-weighted moment tensor

$$
M_{i j}^{(k)}=\sum_{\alpha \beta} \frac{\left\langle 0\left|\hat{Q}_{i}\right| \alpha \beta\right\rangle\left\langle\alpha \beta\left|\hat{Q}_{j}^{+}\right| 0\right\rangle}{\left(E_{\alpha}+E_{\beta}\right)^{k}}
$$

is written in the quasiparticle basis of HFB. In Eq. (2), $|\alpha \beta\rangle$ is a two-quasiparticle wave function and the sum is taken over whole quasiparticle basis for neutrons and protons. $\hat{Q}_{i}$ is the quadupole moment operator, which is either $\hat{Q}_{20}$ or $\hat{Q}_{22}$, and $E_{\alpha}$ denotes the quasiparticle energy. The contours of $M_{i j}^{p C}$ in two-dimension are plotted in Figure 2 for ${ }^{264} \mathrm{Fm}$.

The effective mass $M_{e f f}(s)$ along a particular path $s$ in the collective space is given by $[8,9]$ :

$$
M_{e f f}(s)=\sum_{i j} M_{i j}^{p C} \frac{d Q_{i}}{d s} \frac{d Q_{j}}{d s}
$$

We have tested the numerical accuracy of the quadrupole inertia by utilizing the above expression. First, $M_{e f f}(s)$ is calculated along the negative $\mathrm{Q}_{20}$ axis, which coincides with the oblate deformation axis. Then, the nuclear densities obtained along the negative $\mathrm{Q}_{20}$ axis, are rotated by the Euler angles so that the symmetry axis lies along $\gamma=60^{\circ}$. Since $\gamma=60^{\circ}$ corresponds to oblate shapes in the twodimensional quadrupole deformation plane, the effective mass $M_{e f f}(s)$ along this axis should match the values obtained at $\mathrm{Q}_{20}<0$. This is demonstrated in Figure 3 where the values of $M_{\text {eff }}(s)$ along the two pathways $\left(\gamma=180^{\circ}\right.$ and $\left.\gamma=60^{\circ}\right)$ are shown. 


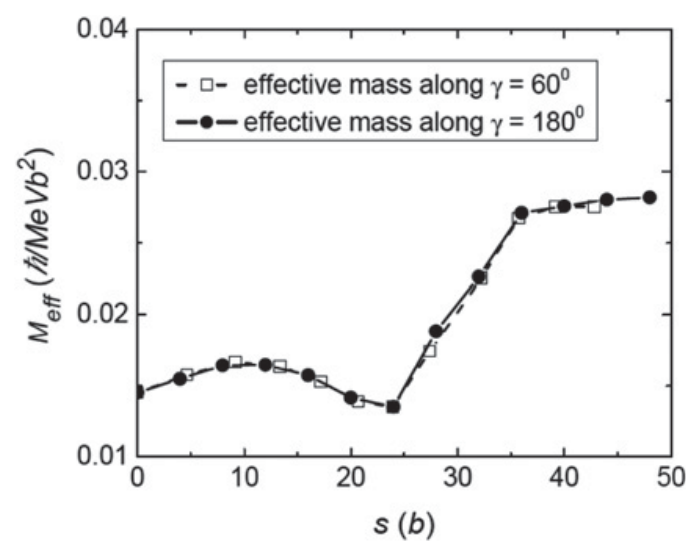

Figure 3. Effective mass $M_{\text {eff }}(s)$ as a function of path length $s$ along $\gamma=180^{\circ}$ and $\gamma=60^{\circ}$.

\section{Action minimization techniques and spontaneous fission half-life}

Here, we describe the numerical techniques to calculate the minimum action path on the twodimensional collective surface. The spontaneous fission half-life $\mathrm{T}_{1 / 2}$ associated with the minimum action path is given by $[8,9]$

$$
T_{1 / 2}=\frac{\ln 2}{n P},
$$

where $n$ is the number of assaults of the nucleus on the fission barrier per unit time and it is often considered to be equal to $10^{20.38} \mathrm{~s}^{-1}$ [9]. The penetration probability $P$ can be estimated from the semiclassical WKB approximation $[8,9]$ :

$$
P=(1+\exp 2 S(L))^{-1},
$$

where $S(L)$ is the action-integral calculated along the fission path $L(s)$ in the multi-dimensional deformation space

$$
S(L)=\int_{s 1}^{s 2}\left[\frac{2}{\hbar} M_{e f f}(s)\left(V(s)-E_{0}\right)\right] d s .
$$

In the above equation, $V(s)$ and $M_{e f f}(s)$ are the potential energy and effective mass along the fission path $L(s)$, respectively. The limits $s 1$ and $s 2$ are classical turning points on $L(s)$, and $d s$ is the element of length, and $E_{0}$ is the ZPE calculated at the ground state configuration. The effective mass $M_{e f f}(s)$ was obtained from the quadrupole mass tensor of Figure 2.

We have calculated the minimum action path by following the dynamic-programming method described in Ref. [8]. Alternatively, the minimum action path can be obtained using the Ritz method [9]. In the latter method, trial paths are expressed as Fourier series of collective coordinates, and the coefficients of different Fourier components are extracted by minimizing the action integral given by Eq. (6). As discussed in Ref. [9], the Ritz method takes much longer computational time than the dynamic-programming method and it is more sensitive to the starting parameter set.

\section{Results}

The minimum action paths obtained with the dynamic programming method and Ritz method are shown in Figure 4 by dashed and dotted lines, respectively. The value of $E_{0}$ is taken to be $1.0 \mathrm{MeV}$ for the sake of a qualitative comparison of different approaches. As seen in Figure 4, the dynamical paths are almost 


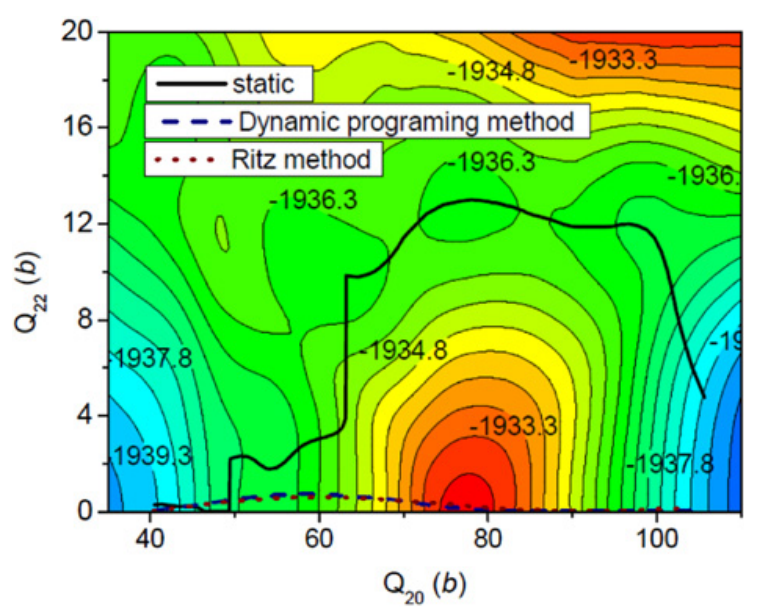

Figure 4. Different spontaneous fission pathways in the collective space $\left(\mathrm{Q}_{20}, \mathrm{Q}_{22}\right)$.

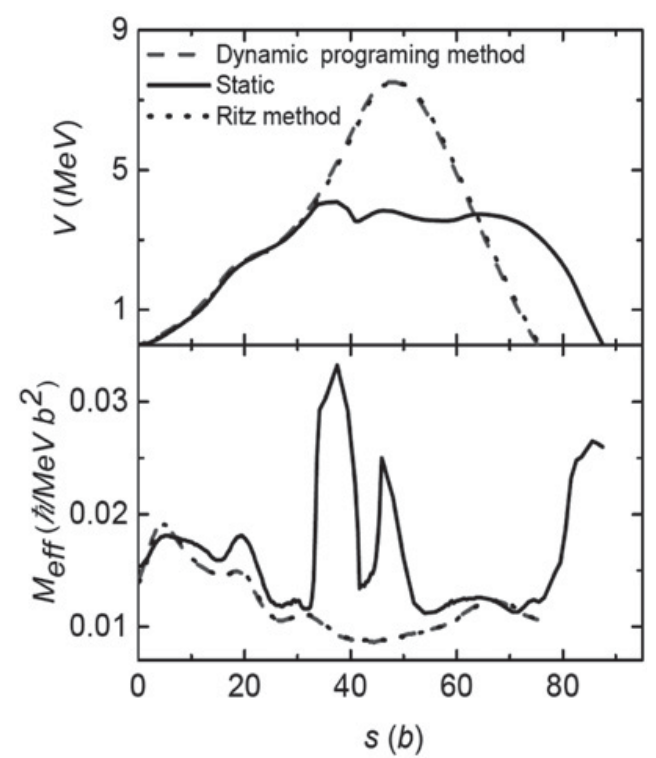

Figure 5. Effective values of potential $V(s)$ (top) and mass parameter $M_{e f f}(s)$ (bottom) along different fission paths in ${ }^{264} \mathrm{Fm}$.

indistinguishable from each other, and this constitutes a stringent test of the method. The static path corresponding to the minimum-energy valley is shown in Figure 4 by a solid line. Clearly, the static path passes goes through strongly triaxial shapes whereas the dynamical paths remain close to the prolate $\left(\mathrm{Q}_{22}=0\right)$ axis. Therefore, a strong dynamical effect-due to variations of the collective inertia with $\mathrm{Q}_{22}$ - prevents the corresponding fission pathways from reaching strongly non-axial shapes. This observation is in accordance with the previously published results in Ref. [10].

To understand better the dynamical effect, in Figure 5 we plot the collective potential $V(s)$ and mass parameter $M_{e f f}(s)$ along different fission pathways. Evidently, the static path traverses a longer distance in the collective space. The dynamical paths are shorter but they go through regions of large 
Table 1. Action integral and spontaneous fission half-life of ${ }^{264} \mathrm{Fm}$ calculated in different methods.

\begin{tabular}{|l|c|c|}
\hline Method & Action integral & $\log \left(T_{1 / 2}\right)(\mathrm{yrs})$ \\
\hline Dynamic programming method & 16.81 & -13.44 \\
Ritz method & 16.80 & -13.45 \\
Static path & 20.78 & -9.99 \\
One-dimension $\left(\mathrm{Q}_{22}=0\right)$ & 16.83 & -13.42 \\
\hline
\end{tabular}

potential energy to avoid areas of large collective mass. This result emphasizes the role of collective mass parameters in determining fission pathways in a multi-dimensional collective space. The values of the action integral and fission half-lives corresponding to different fission pathways are summarized in Table 1 together with the axial (one-dimensional; $\mathrm{Q}_{22}=0$ ) result. One can see that the dynamical results are close to the axial approximation. Also, the outer turning points for the dynamical paths, as shown in Figure 4, remain very close to $\mathrm{Q}_{22}=0$. This suggests that - in the case considered - triaxiality does not contribute significantly to the spontaneous fission lifetime within the perturbative cranking ATDHFB approach.

\section{Summary}

In summary, spontaneous fission lifetimes have been studied within a dynamic approach based on the minimization of the collective action in a two-dimensional collective space of elongation and triaxiality. A strong dynamical effect has been predicted; it offsets the static reduction of the inner barrier by triaxiality. This dynamical effect obviously depends on the nucleus under consideration. In the discussed case of ${ }^{264} \mathrm{Fm}$, the inner barrier is not sufficiently high to counteract the increase of the collective inertia. This observation is consistent with the results of macroscopic-microscopic work [11], which pointed out that the effects of non-axial shapes on the fission process are weakened by the mass tensor. A more detailed study of dynamical effects due to triaxial and reflection asymmetric degrees of freedom is in progress.

This study has been carried out in a collaboration with J. Dobaczewski, A. Baran, and J. Sheikh. Useful discussions with L. Próchniak and A. Staszczak are gratefully acknowledged. This work was supported by the U.S. Department of Energy under Contracts No. DE-FG02-96ER40963 (University of Tennessee), No. DE-FG52-09NA29461 (the Stewardship Science Academic Alliances program), and No. DE-SC0008499 (NUCLEI SciDAC Collaboration). An award of computer time was provided by the National Institute for Computational Sciences (NICS) and the Innovative and Novel Computational Impact on Theory and Experiment (INCITE) program.

\section{References}

[1] M. Baranger and M. Vénéroni, Ann. Phys. 114, 123 (1978); J. Dobaczewski and J. Skalski, Nucl. Phys. A 369, 123 (1981); P. Ring and P. Schuck, The Nuclear Many-Body Problem (SpringerVerlag, Berlin, 1980)

[2] J. W. Negele, Nucl. Phys. A 502, 371 (1989); W. Nazarewicz, Nucl. Phys. A 557, 489c (1993)

[3] A. Baran, J. A. Sheikh, J. Dobaczewski, W. Nazarewicz and A. Staszczak, Phys. Rev. C 84, $054321(2011)$

[4] N. Schunck, J. Dobaczewski, J. McDonnell, W. Satuła, J. A. Sheikh, A. Staszczak, M. Stoitsov, and P. Toivanen, Comput. Phys. Commun. 183, 166 (2012) 
[5] J. Bartel, P. Quentin, M. Brack, C. Guet, and H. B. Håkansson, Nucl. Phys. A 386, 79 (1982)

[6] A. Staszczak, A. Baran and W. Nazarewicz, Phys. Rev. C 87, 024320 (2013)

[7] A. Staszczak, S. Piłat, and K. Pomorski, Nucl. Phys. A 504, 589 (1989); A. Baran, A. Staszczak, J. Dobaczewski, and W. Nazarewicz, Int. J. Mod. Phys. E 16, 443 (2007)

[8] A. Baran, K. Pomorski, A. Łukasiak and A. Sobiczewski, Nucl. Phys. A 361, 83 (1981)

[9] A. Baran, Phys. Lett. B 76, 8 (1978)

[10] J. -P. Delaroche, M. Girod, H. Goutte, and J. Libert Nucl. Phys. A 771, 103 (2006)

[11] R.A. Gherghescu, J. Skalski, Z. Patyk, and A. Sobiczewski, Nucl. Phys. A 651, 237 (1999) 